\title{
Lidil
}

Revue de linguistique et de didactique des langues

$44 \mid 2011$

Langues, minor(is)ations et marginalisations

\section{Christine Develotte, Richard Kern et Marie-Noëlle Lamy, Décrire la conversation en ligne, Le face à face distanciel}

Lyon, ENS Éditions, 2011

Pierre Salam

\section{OpenEdition}

Journals

Édition électronique

URL : http://journals.openedition.org/lidil/3153

DOI : $10.4000 /$ lidil.3153

ISSN : $1960-6052$

Éditeur

UGA Éditions/Université Grenoble Alpes

Édition imprimée

Date de publication : 15 décembre 2011

Pagination : $145-146$

ISBN : 978-2-84310-212-7

ISSN : $1146-6480$

\section{Référence électronique}

Pierre Salam, «Christine Develotte, Richard Kern et Marie-Noëlle Lamy, Décrire la conversation en ligne, Le face à face distanciel », Lidil [En ligne], 44 | 2011, mis en ligne le 15 juin 2013, consulté le 22 septembre 2020. URL : http://journals.openedition.org/lidil/3153 ; DOI : https://doi.org/10.4000/lidil. 3153 


\section{Note de lecture}

Christine Develotte, Richard Kern et Marie-Noëlle Lamy, Décrire la conversation en ligne, Le face à face distanciel, Lyon, ENS

Éditions, 2011, 213 p.

Comme son titre l'indique clairement, l'ouvrage se questionne sur les composantes des échanges multimodaux synchrones. Cependant, l'une des spécificités de ce travail est d'avoir fait appel, en plus des chercheurs reconnus dans le champ de la $\mathrm{CMO}$, à des spécialistes de l'analyse des interactions en face à face. Cette rencontre scientifique permet de réaliser une étude contrastive entre ces deux champs qui partagent plusieurs outils théoriques et méthodologiques.

En outre, ces analystes ont eu à leur disposition une série de corpus « construits » pour ce projet de recherche. En effet, les directeurs ont souhaité reprendre la méthodologie adoptée par l'ouvrage Décrire la conversation de 1987. Ils ont donc fait appel à des étudiants rémunérés qui devaient échanger via un outil de chat multimodal sur une série de thèmes imposés. Les échanges ont été capturés à travers différents outils détaillés dans l'ouvrage, ce qui permet aux chercheurs d'étudier les spécificités linguistiques, phonétiques et mimo-gestuelles des conversations. L'un des objectifs de cette recherche est de poser les bases descriptives de ce genre de communication via l'ordinateur.

L'ouvrage est constitué d'un premier chapitre d'introduction qui permet de définir le champ de recherche et de décrire la méthodologie adoptée par les participants. Les auteurs reviennent sur les différentes définitions de « conversation en ligne » dans la littérature. Par la suite, sept contributions sont présentées dont deux en langue anglaise.

Dans le deuxième chapitre, les auteurs adoptent une approche éthologique compréhensive afin de rendre compte des spécificités des conversations en ligne étudiées. Ils distinguent les principales différences entre les conversations en face à face et celles en mode distanciel, en se basant principalement sur l'analyse des éléments verbaux et posturo-mimogestuels. Cette étude les amène à constater que les difficultés techniques sont rapidement dépassées et que la conversation en ligne amène des conséquences plus variées sur les modalités relationnelles. 
Par la suite, dans le troisième chapitre, l'auteur se focalise sur certains «strategic points » (p. 51) dans les interactions du corpus, à savoir, le début, les interruptions de la parole et la fin de la conversation. L'objectif est de comprendre comment ces mouvements sont compris et accomplis par les participants comme une partie de l'interaction dans un contexte médiatisé par les technologies. Dans la contribution suivante, les auteurs s'interrogent sur les moments où une autre personne fait irruption dans la conversation entre les deux partenaires, pouvant de ce fait causer une rupture de l'échange. S'appuyant sur les analyses de la sémiotique sociale, ils rendent compte de la collaboration multimodale entre les deux interlocuteurs qui cherchent à dépasser cette interruption pour revenir au sujet de leur conversation. Le chapitre cinq est dédié à l'étude de l'influence du contexte spatial de l'interaction en ligne sur l'activité conversationnelle. Après avoir expliqué les raisons qui amènent de nombreuses études à considérer la CMO comme « dé-spatialisée » (p. 96), l'auteur traite du cas particulier des échanges en visioconférence, indiquant ses spécificités spatiales. Il s'intéresse par la suite à certains rôles joués par les contextes spatiaux dans la conversation en ligne ainsi que lorsque l'enjeu des échanges devient un facteur de perturbation ou un cadre déterminant l'activité conversationnelle. L'auteure de la contribution suivante choisit d'étudier la gestion des chevauchements de parole, phénomène d'analyse important en analyse des conversations en face à face dont elle est spécialiste. L'objectif de cette étude est à la fois de faire ressortir les particularités de la gestion de ces chevauchements dans le corpus de conversation en ligne et d'identifier les caractéristiques du dispositif afin de les expliquer. Pour cela, l'auteure commence par une présentation des recherches sur l'analyse des chevauchements que ce soit en face à face ou en distanciel. Par la suite, elle se focalise sur des moments du corpus et schématise certains chevauchements en indiquant comment se passent leur régulation et leur réparation. Se référant à la notion d'ethos, l'avant-dernier auteur analyse la gestion qu'en font les participants aux conversations en ligne. Afin de mettre à jour l'adaptation des interactions aux contraintes imposées par le dispositif, il analyse l'interactivité des regards et des sourires. Cela lui permet de confirmer que la médiation technologique perturbe partiellement l'échange, et il s'interroge sur le rapport entre les différents modes d'énonciations normalement synchrones comme le « dire » et le « montrer».

Le dernier chapitre est un bilan comparatif entre les conversations du corpus de 1987 et celui de 2011. L'auteure, qui a participé aux deux projets de recherche, indique les similarités puis les différences entre les 
deux corpus que ce soit sur le plan de la matérialité de l'interaction, de l'organisation et de la structuration de l'échange, des contenus échangés et de la construction de la relation interpersonnelle.

Pierre Salam

LIDILEM, Université d'Alep (Syrie) 